\title{
Random integrals and correctors in homogenization
}

\author{
Guillaume Bal * Josselin Garnier ${ }^{\dagger} \quad$ Sébastien Motsch ${ }^{\ddagger}$ \\ Vincent Perrier $\S$
}

January 10, 2008

\begin{abstract}
This paper concerns the homogenization of a one-dimensional elliptic equation with oscillatory random coefficients. It is well-known that the random solution to the elliptic equation converges to the solution of an effective medium elliptic equation in the limit of a vanishing correlation length in the random medium. It is also well-known that the corrector to homogenization, i.e., the difference between the random solution and the homogenized solution, converges in distribution to a Gaussian process when the correlations in the random medium are sufficiently short-range. Moreover, the limiting process may be written as a stochastic integral with respect to standard Brownian motion. We generalize the result to a large class of processes with long-range correlations. In this setting, the corrector also converges to a Gaussian random process, which has an interpretation as a stochastic integral with respect to fractional Brownian motion. Moreover, we show that the longer the range of the correlations, the larger is the amplitude of the corrector. Derivations are based on a careful analysis of random oscillatory integrals of processes with long-range correlations. We also make use of the explicit expressions for the solutions to the one-dimensional elliptic equation.
\end{abstract}

\section{Introduction}

Homogenization theory for second-order elliptic equations with highly oscillatory coefficients is well developed, both for periodic and random coefficients; see e.g. 2, 6]. The analysis of correctors, which measure the difference between the heterogeneous solution and the homogenized solution, is more limited.

In the periodic setting, the solution of so-called cell problems allow us to obtain explicit expressions for the correctors. Denoting by $\varepsilon$ the size of the cell of periodicity

*Department of Applied Physics and Applied Mathematics, Columbia University, New York NY, 10027, U.S.A.; gb2030@columbia.edu

${ }^{\dagger}$ Laboratoire de Probabilités et Modèles Aléatoires \& Laboratoire Jacques-Louis Lions, Université Paris VII, 2 Place Jussieu, 75251 Paris Cedex 5, France; garnier@math.jussieu.fr

${ }^{\ddagger}$ Laboratoire MIP, Université Paul Sabatier, 31062 Toulouse Cedex 9, France; motsch@mip.upstlse.fr

§Institut de Mathématiques de Bordeaux and Centre des Lasers Intenses et Applications, Université Bordeaux 1, 351 Cours de la Libération, 33405 Talence Cedex; perrier@math.u-bordeaux1.fr 
of the oscillatory coefficients, the amplitude of the corrector for a second-order equation is typically of order $\varepsilon$ [2, 6].

In the random setting, the situation is complicated by the fact that the local problems are no longer defined on compact cells. And as it turns out, the amplitude of the correctors is no longer of size $\varepsilon$ in general, where $\varepsilon$ now measures the correlation length of the random heterogeneities. Relatively few general estimates are available in the literature on the size of the correctors; see [13]. For the one-dimensional second-order elliptic equation (see (1) below), much more is known because of the availability of explicit expressions for the solutions (see (3) below). The analysis of correctors was taken up in [4], where it is shown that the correctors' amplitude is of order $\sqrt{\varepsilon}$ provided that the random coefficients have sufficiently short-range correlations so that, among other properties, their correlation function is integrable. Moreover, the corrector may be shown to converge in distribution in the space of continuous paths to a Gaussian process, which may be written as a stochastic integral with respect to Brownian motion. This result is recalled in Theorem 2.6 below. The work [4] also proposes error estimates for the corrector in the case of longer-range correlations, when the correlation function of the random coefficients is no longer integrable. The limiting behavior of the corrector is however not characterized.

This paper reconsiders the analysis of correctors for the one-dimensional equation when the correlation function of the random coefficients is no longer integrable, and more precisely takes the form $R(\tau) \sim \tau^{-\alpha}$ as $\tau \rightarrow \infty$ for some $0<\alpha<1$. Longer-range correlations are modeled by smaller values of $\alpha$. A prototypical example of a continuous, stationary process with long-range correlation is a normalized Gaussian process $g_{x}$ with a correlation function $R_{g}(\tau)=\mathbb{E}\left\{g_{x} g_{x+\tau}\right\}$ that decays as $\tau^{-\alpha}$. The random coefficients for the elliptic equation we consider in this paper are mean zero stationary processes that can be written as $\varphi(x)=\Phi\left(g_{x}\right)$, where $\Phi$ is a bounded function. Under appropriate assumptions on $\Phi$, the correlation function of $\varphi$ also decays as $\tau^{-\alpha}$ as $\tau \rightarrow \infty$.

For the random coefficients described above, we show that the corrector to homogenization has an amplitude of order $\varepsilon^{\alpha}$ and converges in distribution to a Gaussian process that may be represented as a stochastic integral with respect to a fractional Brownian motion $W_{t}^{H}$ with Hurst index $H=1-\frac{\alpha}{2}$. The limit $\alpha \rightarrow 1$ thus converges to the case of integrable correlation function. Note however that in the limit of very long-range correlations as $\alpha \rightarrow 0$, the influence of the corrector becomes more and more important. The main tool in our derivation is a careful convergence analysis in distribution of oscillatory integrals of the form $\int_{0}^{1} K(x, t) \varepsilon^{-\frac{\alpha}{2}} \varphi\left(\frac{t}{\varepsilon}\right) d t$ to a stochastic integral with respect to fractional Brownian motion, where $K(x, t)$ is a known kernel and $\varphi(t)$ is a random process with long-range correlations. This analysis extends weak convergence results obtained for sums of random variables or for integrals of random processes with long-range correlations in [12, 9].

The paper is structured as follows. Section 2 presents the heterogeneous and homogeneous one-dimensional elliptic equations and describes our hypotheses on the random coefficients. The section concludes by a statement of Theorem 2.5, which is our main result. The analysis of random oscillatory integrals of the form $\int_{0}^{1} F(t) \varepsilon^{-\frac{\alpha}{2}} \varphi\left(\frac{t}{\varepsilon}\right) d t$ is carried out in section 3. Theorem [3.1 shows their convergence to stochastic integrals with respect to fractional Brownian motion $W_{t}^{H}$. Section 4 shows how the results of section 3 extend to the analysis of the processes of the form $\int_{0}^{1} K(x, t) \varepsilon^{-\frac{\alpha}{2}} \varphi\left(\frac{t}{\varepsilon}\right) d t$ that arise in 
the analysis of the correctors to homogenization. The convergence in distribution in the space of continuous paths of such processes to a Gaussian processes is summarized in theorem 4.1. The theoretical results are backed up by numerical simulations in section 5. After a detailed description of the construction of random processes with given long-range correlations, we demonstrate the convergence of random oscillatory integrals and of homogenization correctors to their appropriate limits as stochastic integrals with respect to fractional Brownian motion. Some concluding remarks are given in section [7]

\section{One-dimensional homogenization}

\subsection{Homogenization problem}

We are interested in the solution to the following elliptic equation with random coefficients

$$
\begin{aligned}
& -\frac{d}{d x}\left(a\left(\frac{x}{\varepsilon}, \omega\right) \frac{d}{d x} u^{\varepsilon}\right)=f(x), \quad 0 \leq x \leq 1, \quad \omega \in \Omega, \\
& u^{\varepsilon}(0, \omega)=0, \quad u^{\varepsilon}(1, \omega)=q .
\end{aligned}
$$

Here $a(x, \omega)$ is a stationary ergodic random process such that $0<a_{0} \leq a(x, \omega) \leq a_{0}^{-1}$ a.e. for $(x, \omega) \in(0,1) \times \Omega$, where $(\Omega, \mathcal{F}, \mathbb{P})$ is an abstract probability space. The source term $f \in W^{-1, \infty}(0,1)$ and $q \in \mathbb{R}$. Classical theories for elliptic equations then show the existence of a unique solution $u(\cdot, \omega) \in H^{1}(0,1) \mathbb{P}$-a.s.

As the scale of the micro-structure $\varepsilon$ converges to 0 , the solution $u^{\varepsilon}(x, \omega)$ converges $\mathbb{P}$-a.s. weakly in $H^{1}(0,1)$ to the deterministic solution $\bar{u}$ of the homogenized equation

$$
\begin{aligned}
& -\frac{d}{d x}\left(a^{*} \frac{d}{d x} \bar{u}\right)=f(x), \quad 0 \leq x \leq 1, \\
& \bar{u}(0)=0, \quad \bar{u}(1)=q .
\end{aligned}
$$

The effective diffusion coefficient is given by $a^{*}=\left(\mathbb{E}\left\{a^{-1}(0, \cdot)\right\}\right)^{-1}$, where $\mathbb{E}$ is mathematical expectation with respect to $\mathbb{P}$. See e.g. [6, 7, 10].

The above one-dimensional boundary value problems admit explicit solutions. Introducing $a^{\varepsilon}(x)=a\left(\frac{x}{\varepsilon}\right)$ and $F(x)=\int_{0}^{x} f(y) d y$, we have:

$$
\begin{aligned}
u^{\varepsilon}(x, \omega)=c^{\varepsilon}(\omega) \int_{0}^{x} \frac{1}{a^{\varepsilon}(y, \omega)} d y-\int_{0}^{x} \frac{F(y)}{a^{\varepsilon}(y, \omega)} d y, \quad c^{\varepsilon}(\omega)=\frac{q+\int_{0}^{1} \frac{F(y)}{a^{\varepsilon}(y, \omega)} d y}{\int_{0}^{1} \frac{1}{a^{\varepsilon}(y, \omega)} d y} \\
\bar{u}(x)=c^{*} \frac{x}{a^{*}}-\int_{0}^{x} \frac{F(y)}{a^{*}} d y, \quad c^{*}=a^{*} q+\int_{0}^{1} F(y) d y .
\end{aligned}
$$

Our aim is to characterize the behavior of $u^{\varepsilon}-\bar{u}$ as $\varepsilon \rightarrow 0$.

\subsection{Hypothesis on the random process $a$}

In order to characterize the behavior of the corrector $u^{\varepsilon}-\bar{u}$ as $\varepsilon \rightarrow 0$, we need additional assumptions on the random process $a(x, \omega)$. Let us define the mean zero stationary 
random process

$$
\varphi(x, \omega)=\frac{1}{a(x, \omega)}-\frac{1}{a^{*}} .
$$

Hypothesis [H]. We assume that $\varphi$ is of the form

$$
\varphi(x)=\Phi\left(g_{x}\right),
$$

where $\Phi$ is a bounded function such that

$$
\int \Phi(g) e^{-\frac{g^{2}}{2}} d g=0
$$

and $g_{x}$ is a stationary Gaussian process with mean zero and variance one. The autocorrelation function of $g$ :

$$
R_{g}(\tau)=\mathbb{E}\left\{g_{x} g_{x+\tau}\right\},
$$

is assumed to have a heavy tail of the form

$$
R_{g}(\tau) \sim \kappa_{g} \tau^{-\alpha} \text { as } \tau \rightarrow \infty
$$

where $\kappa_{g}>0$ and $\alpha \in(0,1)$.

Remark 2.1. This hypothesis is satisfied by a large class of random coefficients. For instance, if we take $\Phi=\operatorname{sgn}$, then $\varphi$ models a two-component medium. If we take $\Phi=$ tanh or arctan, then $\varphi$ models a continuous medium with bounded variations.

The autocorrelation function of the random process $a$ has a heavy tail, as stated in the following proposition.

Proposition 2.2. The process $\varphi$ defined by (6) is a stationary random process with mean zero and variance $V_{2}$. Its autocorrelation function

$$
R(\tau)=\mathbb{E}\{\varphi(x) \varphi(x+\tau)\}
$$

has a heavy tail of the form

$$
R(\tau) \sim \kappa \tau^{-\alpha} \text { as } \tau \rightarrow \infty
$$

where $\kappa=\kappa_{g} V_{1}^{2}$,

$$
\begin{aligned}
& V_{1}=\mathbb{E}\left\{g_{0} \Phi\left(g_{0}\right)\right\}=\frac{1}{\sqrt{2 \pi}} \int g \Phi(g) e^{-\frac{g^{2}}{2}} d g, \\
& V_{2}=\mathbb{E}\left\{\Phi^{2}\left(g_{0}\right)\right\}=\frac{1}{\sqrt{2 \pi}} \int \Phi^{2}(g) e^{-\frac{g^{2}}{2}} d g .
\end{aligned}
$$

Proof. The fact that $\varphi$ is a stationary random process with mean zero and variance $V_{2}$ is straightforward in view of the definition of $\varphi$. In particular, Eq. (17) implies that $\varphi$ has mean zero.

For any $x, \tau$, the vector $\left(g_{x}, g_{x+\tau}\right)^{T}$ is a Gaussian random vector with mean $(0,0)^{T}$ and $2 \times 2$ covariance matrix:

$$
C=\left(\begin{array}{cc}
1 & R_{g}(\tau) \\
R_{g}(\tau) & 1
\end{array}\right)
$$


Therefore the autocorrelation function of the process $\varphi$ is

$$
\begin{aligned}
R(\tau) & =\mathbb{E}\left\{\Phi\left(g_{x}\right) \Phi\left(g_{x+\tau}\right)\right\}=\frac{1}{2 \pi \sqrt{\operatorname{det} C}} \iint \Phi\left(g_{1}\right) \Phi\left(g_{2}\right) \exp \left(-\frac{g^{T} C^{-1} g}{2}\right) d^{2} g \\
& =\frac{1}{2 \pi \sqrt{1-R_{g}^{2}(\tau)}} \iint \Phi\left(g_{1}\right) \Phi\left(g_{2}\right) \exp \left(-\frac{g_{1}^{2}+g_{2}^{2}-2 R_{g}(\tau) g_{1} g_{2}}{2\left(1-R_{g}^{2}(\tau)\right)}\right) d g_{1} d g_{2} .
\end{aligned}
$$

For large $\tau$, the coefficient $R_{g}(\tau)$ is small and we can expand the value of the double integral in powers of $R_{g}(\tau)$, which gives the autocorrelation function of $\varphi$.

To simplify notation, we no longer write the $\omega$-dependence explicitly and we define $\varphi^{\varepsilon}(x)=\varphi\left(\frac{x}{\varepsilon}\right)$.

\subsection{Analysis of the corrector}

The purpose of this section is to show that the error term $u^{\varepsilon}-\bar{u}$ has two different contributions: integrals of random processes with long term memory effects and lowerorder terms. The analysis of integrals of the random processes with long term memory effects is carried out in the next sections. The following lemma, whose proof can be found in Section 4.2, provides an estimate for the magnitude of these integrals.

Lemma 2.3. Let $\varphi(x)$ be a mean zero stationary random process of the form (6)). There exists $K>0$ such that, for any $F \in L^{\infty}(0,1)$, we have

$$
\sup _{x \in[0,1]} \mathbb{E}\left\{\left|\int_{0}^{x} \varphi^{\varepsilon}(t) F(t) d t\right|^{2}\right\} \leq K\|F\|_{\infty}^{2} \varepsilon^{\alpha} .
$$

As a corollary, we obtain the following:

Corollary 2.4. Let $\varphi(x)$ be a mean zero stationary random process of the form (6) and let $f \in W^{-1, \infty}(0,1)$. The solutions $u^{\varepsilon}$ of (3) and $\bar{u}$ of (41) verify that:

$$
u^{\varepsilon}(x)-\bar{u}(x)=-\int_{0}^{x} \varphi^{\varepsilon}(y) F(y) d y+\left(c^{\varepsilon}-c^{*}\right) \frac{x}{a^{*}}+c^{*} \int_{0}^{x} \varphi^{\varepsilon}(y) d y+r^{\varepsilon}(x),
$$

where

$$
\sup _{x \in[0,1]} \mathbb{E}\left\{\left|r^{\varepsilon}(x)\right|\right\} \leq K \varepsilon^{\alpha},
$$

for some $K>0$. Similarly, we have that

$$
c^{\varepsilon}-c^{*}=a^{*} \int_{0}^{1}\left(F(y)-\int_{0}^{1} F(z) d z-a^{*} q\right) \varphi^{\varepsilon}(y) d y+\rho^{\varepsilon},
$$

where

$$
\mathbb{E}\left\{\left|\rho^{\varepsilon}\right|\right\} \leq K \varepsilon^{\alpha},
$$

for some $K>0$. 
Proof. We first establish the estimate for $c^{\varepsilon}-c$. We write

$$
c^{\varepsilon}-c^{*}=\frac{\int_{0}^{1} F(y)\left(\frac{1}{a^{\varepsilon}(y)}-\frac{1}{a^{*}}\right) d y}{\int_{0}^{1} \frac{1}{a^{\varepsilon}(y)} d y}+\left(q+\frac{1}{a^{*}} \int_{0}^{1} F(y) d y\right)\left(\frac{1}{\int_{0}^{1} \frac{1}{a^{\varepsilon}(y)} d y}-\frac{1}{\frac{1}{a^{*}}}\right),
$$

which gives (16) with

$$
\rho^{\varepsilon}=\frac{a^{*}}{\int_{0}^{1} \frac{1}{a^{\varepsilon}(y)} d y}\left[\left(a^{*} q+\int_{0}^{1} F(y) d y\right)\left(\int_{0}^{1} \varphi^{\varepsilon}(y) d y\right)^{2}-\int_{0}^{1} F(y) \varphi^{\varepsilon}(y) d y \int_{0}^{1} \varphi^{\varepsilon}(y) d y\right] .
$$

Since $\int_{0}^{1} \frac{1}{a^{\varepsilon}(y)} d y$ is bounded from below a.e. by a positive constant $a_{0}$, we deduce from Lemma [2.3] and the Cauchy-Schwarz estimate that $\mathbb{E}\left\{\left|\rho^{\varepsilon}\right|\right\} \leq K \varepsilon^{\alpha}$. The analysis of $u^{\varepsilon}-\bar{u}$ follows along the same lines. We write

$$
u^{\varepsilon}(x)-\bar{u}(x)=c^{\varepsilon} \int_{0}^{x} \frac{1}{a^{\varepsilon}(y)} d y-\int_{0}^{x} \frac{F(y)}{a^{\varepsilon}(y)} d y-c^{*} \frac{x}{a^{*}}+\int_{0}^{x} \frac{F(y)}{a^{*}} d y,
$$

which gives (14) with

$$
r^{\varepsilon}(x)=\left(c^{\varepsilon}-c^{*}\right) \int_{0}^{x} \varphi^{\varepsilon}(y) d y=r_{1}^{\varepsilon}(x)+r_{2}^{\varepsilon}(x),
$$

where we have defined

$$
\begin{aligned}
& r_{1}^{\varepsilon}(x)=\left[a^{*} \int_{0}^{1}\left(F(y)-\int_{0}^{1} F(z) d z-a^{*} q\right) \varphi^{\varepsilon}(y) d y\right]\left[\int_{0}^{x} \varphi^{\varepsilon}(y) d y\right], \\
& r_{2}^{\varepsilon}(x)=\rho^{\varepsilon}\left[\int_{0}^{x} \varphi^{\varepsilon}(y) d y\right] .
\end{aligned}
$$

The Cauchy-Schwarz estimate and Lemma 2.3 give that $\mathbb{E}\left\{\left|r_{1}^{\varepsilon}(x)\right|\right\} \leq K \varepsilon^{\alpha}$. Besides, $\varphi^{\varepsilon}$ is bounded by $\|\Phi\|_{\infty}$, so $\left|r_{2}^{\varepsilon}(x)\right| \leq\|\Phi\|_{\infty}\left|\rho^{\varepsilon}\right|$. The estimate on $\rho^{\varepsilon}$ then shows that $\mathbb{E}\left\{\left|r_{2}^{\varepsilon}(x)\right|\right\} \leq K \varepsilon^{\alpha}$.

The previous corollary shows that the error term $u^{\varepsilon}(x)-\bar{u}(x)$ involves integrals of random coefficients of order $\varepsilon^{\alpha / 2}$ up to lower-order terms of order $\varepsilon^{\alpha}$.

\subsection{Homogenization theorem}

The results we obtain in the following sections allow for the following characterization of the correctors.

Theorem 2.5. Let $u^{\varepsilon}$ and $\bar{u}$ be the solutions in (3) and (44), respectively, and let $\varphi(x)$ be a mean zero stationary random process of the form (6). Then $u^{\varepsilon}-\bar{u}$ is a random process in $\mathcal{C}(0,1)$, the space of continuous functions on $[0,1]$. We have the following convergence in distribution in the space of continuous functions $\mathcal{C}(0,1)$ :

$$
\frac{u^{\varepsilon}(x)-\bar{u}(x)}{\varepsilon^{\frac{\alpha}{2}}} \stackrel{\text { distribution }}{\longrightarrow} \sqrt{\frac{\kappa}{H(2 H-1)}} \mathcal{U}^{H}(x),
$$


where

$$
\begin{aligned}
\mathcal{U}^{H}(x) & =\int_{\mathbb{R}} K(x, t) d W_{t}^{H}, \\
K(x, t) & =\mathbf{1}_{[0, x]}(t)\left(c^{*}-F(t)\right)+x\left(F(t)-\int_{0}^{1} F(z) d z-a^{*} q\right) \mathbf{1}_{[0,1]}(t) .
\end{aligned}
$$

Here $\mathbf{1}_{[0, x]}$ is the characteristic function of the set $[0, x]$ and $W_{t}^{H}$ is a fractional Brownian motion with Hurst index $H=1-\frac{\alpha}{2}$.

The proof of this theorem is postponed to Section 4.3 For the convenience of the reader, we present a rapid overview of the integration theory with respect to a fractional Brownian motion. The fractional Brownian motion $W_{t}^{H}$ is a mean zero Gaussian process with autocorrelation function

$$
\mathbb{E}\left\{W_{t}^{H} W_{s}^{H}\right\}=\frac{1}{2}\left(|t|^{2 H}+|s|^{2 H}-|s-t|^{2 H}\right) .
$$

In particular, the variance of $W_{t}^{H}$ is $\mathbb{E}\left\{\left|W_{t}^{H}\right|^{2}\right\}=|t|^{2 H}$. The increments of $W_{t}^{H}$ are stationary but not independent for $H \neq \frac{1}{2}$. Moreover, $W_{t}^{H}$ admits the following spectral representation

$$
W_{t}^{H}=\frac{1}{2 \pi C(H)} \int_{\mathbb{R}} \frac{e^{i \xi t}-1}{i \xi|\xi|^{H-\frac{1}{2}}} d \hat{W}(\xi), \quad t \in \mathbb{R}
$$

where

$$
C(H)=\left(\frac{1}{2 H \sin (\pi H) \Gamma(2 H)}\right)^{1 / 2},
$$

and $\hat{W}$ is the Fourier transform of a standard Brownian motion $W$, that is, a complex Gaussian measure such that:

$$
\mathbb{E}\left\{d \hat{W}(\xi) \overline{d \hat{W}\left(\xi^{\prime}\right)}\right\}=2 \pi \delta\left(\xi-\xi^{\prime}\right) d \xi d \xi^{\prime} .
$$

Note that the constant $C(H)$ is defined such that $\mathbb{E}\left\{\left(W_{1}^{H}\right)^{2}\right\}=1$ because we have that

$$
C^{2}(H)=\frac{1}{2 \pi} \int_{\mathbb{R}} \frac{\left|e^{i \xi}-1\right|^{2}}{|\xi|^{2 H+1}} d \xi .
$$

The integral with respect to the fractional Brownian motion is defined for a large class of deterministic functions $F$ (see [11] for an extensive review). Functions in $L^{1}(\mathbb{R}) \cap L^{2}(\mathbb{R})$ are in the class of integrable functions when $H \in\left(\frac{1}{2}, 1\right)$, which is the range of values of $H$ considered in Theorem 2.5. Using the representation (22), we have, in distribution, for any $F \in L^{1}(\mathbb{R}) \cap L^{2}(\mathbb{R})$,

$$
\int_{\mathbb{R}} F(t) d W_{t}^{H}=\frac{1}{2 \pi C(H)} \int_{\mathbb{R}} \frac{\hat{F}(\xi)}{|\xi|^{H-\frac{1}{2}}} d \hat{W}(\xi),
$$

where the Fourier transform $\hat{F}(\xi)$ of a function $F(t)$ is defined by

$$
\hat{F}(\xi)=\int_{\mathbb{R}} e^{i t \xi} F(t) d t .
$$


When $F, G \in L^{1}(\mathbb{R}) \cap L^{2}(\mathbb{R})$, the random vector $\left(\int_{\mathbb{R}} F(t) d W_{t}^{H}, \int_{\mathbb{R}} G(t) d W_{t}^{H}\right)$ is a mean zero Gaussian vector with covariance

$$
\mathbb{E}\left\{\int_{\mathbb{R}} F(t) d W_{t}^{H} \int_{\mathbb{R}} G(t) d W_{t}^{H}\right\}=\frac{1}{2 \pi C(H)^{2}} \int_{\mathbb{R}} \frac{\hat{F}(\xi) \overline{\hat{G}(\xi)}}{|\xi|^{2 H-1}} d \xi .
$$

As a consequence, in Theorem 2.5, the limit process $\mathcal{U}^{H}(x)$ is a mean zero Gaussian process with autocorrelation function given by

$$
\mathbb{E}\left\{\mathcal{U}^{H}(x) \mathcal{U}^{H}(y)\right\}=\frac{1}{2 \pi C(H)^{2}} \int_{\mathbb{R}} \frac{\hat{K}(x, \xi) \overline{\hat{K}(y, \xi)}}{|\xi|^{2 H-1}} d \xi
$$

where $\hat{K}(x, \xi)$ is the Fourier transform with respect to $t$ of $K(x, t)$. Finally, using the notation

$$
\int_{0}^{x} F(s) d W_{t}^{H}=\int_{\mathbb{R}} \mathbf{1}_{[0, x]}(s) F(s) d W_{t}^{H},
$$

the limit process $\mathcal{U}^{H}(x)$ defined by (19) can also be written as

$$
\mathcal{U}^{H}(x)=c^{*} W_{x}^{H}-\int_{0}^{x} F(t) d W_{t}^{H}+x \int_{0}^{1} F(t) d W_{t}^{H}-x\left(\int_{0}^{1} F(z) d z-a^{*} q\right) W_{1}^{H} .
$$

The result of Theorem 2.5 should be contrasted with the convergence result for processes with short term memory:

Theorem 2.6. Let $u^{\varepsilon}$ and $\bar{u}$ be as in Theorem 2.5 and let $\varphi(x)$ be a mean zero stationary random process of the form (6). If the correlation function $R_{g}$ of $g$ is integrable (instead of being equivalent to $\tau^{-\alpha}$ at infinity), then $R$ is also integrable. The corrector $u^{\varepsilon}-\bar{u}$ is a random process in $\mathcal{C}[0,1]$ and we have the following convergence in $\mathcal{C}(0,1)$

$$
\frac{u^{\varepsilon}(x)-\bar{u}(x)}{\sqrt{\varepsilon}} \stackrel{\text { distribution }}{\longrightarrow}\left(2 \int_{0}^{\infty} R(\tau) d \tau\right)^{1 / 2} \mathcal{U}(x),
$$

where

$$
\mathcal{U}(x)=\int_{\mathbb{R}} K(x, t) d W_{t},
$$

$K(x, t)$ is given by (20), and $W_{t}$ is standard Brownian motion.

The limit process $\mathcal{U}(x)$ can also be written in the form

$$
\mathcal{U}(x)=c^{*} W_{x}-\int_{0}^{x} F(t) d W_{t}+x \int_{0}^{1} F(t) d W_{t}-x\left(\int_{0}^{1} F(z) d z-a^{*} q\right) W_{1} .
$$

Such a result is based on standard techniques of approximation of oscillatory integrals [8] and was first derived in 4 . In the next sections, we focus our attention to the analysis of random variables or random processes defined in terms of integrals of random processes with long-term memory. 


\section{Convergence of random integrals}

In this section, we aim at proving the following theorem.

Theorem 3.1. Let $\varphi$ be of the form ([6) and let $F \in L^{1}(\mathbb{R}) \cap L^{\infty}(\mathbb{R})$. We define the mean zero random variable $M_{F}^{\varepsilon}$ by

$$
M_{F}^{\varepsilon}=\varepsilon^{-\frac{\alpha}{2}} \int_{\mathbb{R}} \varphi^{\varepsilon}(t) F(t) d t .
$$

Then the random variable $M_{F}^{\varepsilon}$ converges in distribution as $\varepsilon \rightarrow 0$ to the mean zero Gaussian random variable $M_{F}^{0}$ defined by

$$
M_{F}^{0}=\sqrt{\frac{\kappa}{H(2 H-1)}} \int_{\mathbb{R}} F(t) d W_{t}^{H},
$$

where $W_{t}^{H}$ is a fractional Brownian motion with Hurst index $H=1-\frac{\alpha}{2}$.

The limit random variable $M_{F}^{0}$ is a Gaussian random variable with mean zero and variance

$$
\mathbb{E}\left\{\left|M_{F}^{0}\right|^{2}\right\}=\frac{\kappa}{H(2 H-1)} \times \frac{1}{2 \pi C(H)^{2}} \int_{\mathbb{R}} \frac{|\hat{F}(\xi)|^{2}}{|\xi|^{2 H-1}} d \xi
$$

In order to prove Theorem 3.1, we show in Subsection 3.1] that the variance of $M_{F}^{\varepsilon}$ converges to the variance of $M_{F}^{0}$ as $\varepsilon \rightarrow 0$. In Subsection 3.2. we prove convergence in distribution by using the Gaussian property of the underlying process $g_{x}$.

\subsection{Convergence of the variances}

We begin with a key technical lemma.

Lemma 3.2. 1. There exist $T, K>0$ such that the autocorrelation function $R(\tau)$ of the process $\varphi$ satisfies

$$
\left|R(\tau)-V_{1}^{2} R_{g}(\tau)\right| \leq K R_{g}(\tau)^{2}, \quad \text { for all } \quad|\tau| \geq T .
$$

2. There exist $T, K$ such that

$$
\left|\mathbb{E}\left\{g_{x} \Phi\left(g_{x+\tau}\right)\right\}-V_{1} R_{g}(\tau)\right| \leq K R_{g}^{2}(\tau) \quad \text { for all } \quad|\tau| \geq T .
$$

Proof. The first point is a refinement of what we proved in Proposition 2.2, we found that the autocorrelation function of the process $\varphi$ is

$$
R(\tau)=\frac{1}{2 \pi \sqrt{1-R_{g}^{2}(\tau)}} \iint \Phi\left(g_{1}\right) \Phi\left(g_{2}\right) \exp \left(-\frac{g_{1}^{2}+g_{2}^{2}-2 R_{g}(\tau) g_{1} g_{2}}{2\left(1-R_{g}^{2}(\tau)\right)}\right) d g_{1} d g_{2} .
$$

For large $\tau$, the coefficient $R_{g}(\tau)$ is small and we can expand the value of the double integral in powers of $R_{g}(\tau)$, which gives the result of the first item. The proof of the second item follows along the same lines. We first write

$$
\mathbb{E}\left\{g_{x} \Phi\left(g_{x+\tau}\right)\right\}=\frac{1}{2 \pi \sqrt{1-R_{g}^{2}(\tau)}} \iint g_{1} \Phi\left(g_{2}\right) \exp \left(-\frac{g_{1}^{2}+g_{2}^{2}-2 R_{g}(\tau) g_{1} g_{2}}{2\left(1-R_{g}^{2}(\tau)\right)}\right) d g_{1} d g_{2},
$$


and we expand the value of the double integral in powers of $R_{g}(\tau)$.

For $F \in L^{1}(\mathbb{R}) \cap L^{\infty}(\mathbb{R})$, we define the mean zero random variable $M_{F}^{\varepsilon, g}$ by

$$
M_{F}^{\varepsilon, g}=\varepsilon^{-\frac{\alpha}{2}} \int_{\mathbb{R}} g_{\frac{t}{\varepsilon}} F(t) d t .
$$

The purpose of this subsection is to determine the limits of the variances of the variables $M_{F}^{\varepsilon}$ and $M_{F}^{\varepsilon, g}$.

Lemma 3.3. Let $F \in L^{1}(\mathbb{R}) \cap L^{\infty}(\mathbb{R})$ and let $g_{x}$ be the Gaussian random process described in Hypothesis [H], Then

$$
\lim _{\varepsilon \rightarrow 0} \mathbb{E}\left\{\left|M_{F}^{\varepsilon, g}\right|^{2}\right\}=\frac{\kappa_{g} 2^{-\alpha} \Gamma\left(\frac{1-\alpha}{2}\right)}{\sqrt{\pi} \Gamma\left(\frac{\alpha}{2}\right)} \int_{\mathbb{R}} \frac{|\hat{F}(\xi)|^{2}}{|\xi|^{1-\alpha}} d \xi .
$$

Proof. We write the square of the integral as a double integral, which gives

$$
\mathbb{E}\left\{\left|\int_{\mathbb{R}} F(y) g_{\frac{y}{\varepsilon}} d y\right|^{2}\right\}=\int_{\mathbb{R}^{2}} R_{g}\left(\frac{y-z}{\varepsilon}\right) F(y) F(z) d y d z .
$$

This implies the estimate

$$
\begin{aligned}
& \left|\mathbb{E}\left\{\left|M_{F}^{\varepsilon, g}\right|^{2}\right\}-\int_{\mathbb{R}^{2}} \frac{\kappa_{g}}{|y-z|^{\alpha}} F(y) F(z) d y d z\right| \\
& \quad \leq \int_{\mathbb{R}^{2}}\left|\varepsilon^{-\alpha} R_{g}\left(\frac{y-z}{\varepsilon}\right)-\frac{\kappa_{g}}{|y-z|^{\alpha}}\right||F(y)||F(z)| d y d z .
\end{aligned}
$$

By (8), for any $\delta>0$, there exists $T_{\delta}$ such that, for all $|\tau| \geq T_{\delta}$,

$$
\left|R_{g}(\tau)-\kappa_{g} \tau^{-\alpha}\right| \leq \delta \tau^{-\alpha}
$$

We decompose the integration domain into three subdomains $D_{1}, D_{2}$, and $D_{3}$ :

$$
\begin{aligned}
& D_{1}=\left\{(y, z) \in \mathbb{R}^{2},|y-z| \leq T_{\delta} \varepsilon\right\}, \\
& D_{2}=\left\{(y, z) \in \mathbb{R}^{2}, T_{\delta} \varepsilon<|y-z| \leq 1\right\}, \\
& D_{3}=\left\{(y, z) \in \mathbb{R}^{2}, 1<|y-z|\right\} .
\end{aligned}
$$

First,

$$
\begin{aligned}
\int_{D_{1}} \mid & \varepsilon^{-\alpha} R_{g}\left(\frac{y-z}{\varepsilon}\right)-\frac{\kappa_{g}}{|y-z|^{\alpha}}|| F(y)|| F(z) \mid d y d z \\
& \leq \int_{D_{1}}\left|\varepsilon^{-\alpha} R_{g}\left(\frac{y-z}{\varepsilon}\right)\right||F(y)||F(z)| d y d z+\int_{D_{1}} \kappa_{g}|y-z|^{-\alpha}|F(y)||F(z)| d y d z \\
& \leq 2 \varepsilon^{-\alpha}\left\|R_{g}\right\|_{\infty} \int_{\mathbb{R}} \int_{0}^{T_{\delta} \varepsilon}|F(y+z)| d y|F(z)| d z+2 \kappa_{g} \int_{\mathbb{R}} \int_{0}^{T_{\delta} \varepsilon} y^{-\alpha}|F(y+z)| d y|F(z)| d z \\
& \leq 2 \varepsilon^{-\alpha}\left\|R_{g}\right\|_{\infty}\|F\|_{\infty}\|F\|_{1} \int_{0}^{T_{\delta} \varepsilon} d y+2 \kappa_{g}\|F\|_{\infty}\|F\|_{1} \int_{0}^{T_{\delta} \varepsilon} y^{-\alpha} d y \\
& \leq\|F\|_{\infty}\|F\|_{1}\left(2 T_{\delta} R_{g}(0)+\frac{2 \kappa_{g} T_{\delta}^{1-\alpha}}{1-\alpha}\right) \varepsilon^{1-\alpha},
\end{aligned}
$$


where we have used the fact that $R_{g}(\tau)$ is maximal at $\tau=0$, and the value of the maximum is equal to the variance of $g$. Second,

$$
\begin{aligned}
\int_{D_{2}}\left|\varepsilon^{-\alpha} R_{g}\left(\frac{y-z}{\varepsilon}\right)-\frac{\kappa_{g}}{|y-z|^{\alpha}}\right||F(y)||F(z)| d y d z & \leq \delta \int_{D_{2}}|y-z|^{-\alpha}|F(y) \| F(z)| d y d z \\
& \leq 2 \delta\|F\|_{\infty}\|F\|_{1} \int_{T_{\delta} \varepsilon}^{1} y^{-\alpha} d y \\
& \leq \frac{2 \delta\|F\|_{\infty}\|F\|_{1}}{1-\alpha},
\end{aligned}
$$

and finally

$$
\begin{aligned}
\int_{D_{3}}\left|\varepsilon^{-\alpha} R_{g}\left(\frac{y-z}{\varepsilon}\right)-\frac{\kappa_{g}}{|y-z|^{\alpha}}\right||F(y)||F(z)| d y d z & \leq \delta \int_{D_{3}}|y-z|^{-\alpha}|F(y)||F(z)| d y d z \\
& \leq \delta \int_{D_{3}}|F(y) \| F(z)| d y d z \\
& \leq \delta\|F\|_{1}^{2} .
\end{aligned}
$$

Therefore, there exists $K>0$ such that

$$
\limsup _{\varepsilon \rightarrow 0}\left|\mathbb{E}\left\{\left|M_{F}^{\varepsilon, g}\right|^{2}\right\}-\int_{\mathbb{R}^{2}} \frac{\kappa_{g}}{|y-z|^{\alpha}} F(y) F(z) d y d z\right| \leq K\left(\|F\|_{\infty}^{2}+\|F\|_{1}^{2}\right) \delta .
$$

Since this holds true for any $\delta>0$, we get

$$
\lim _{\varepsilon \rightarrow 0}\left|\mathbb{E}\left\{\left|M_{F}^{\varepsilon, g}\right|^{2}\right\}-\int_{\mathbb{R}^{2}} \frac{\kappa_{g}}{|y-z|^{\alpha}} F(y) F(z) d y d z\right|=0 .
$$

We recall that the Fourier transform of the function $|x|^{-\alpha}$ is

$$
\widehat{|x|^{-\alpha}}(\xi)=c_{\alpha}|\xi|^{\alpha-1}, \quad c_{\alpha}=\int_{\mathbb{R}} \frac{e^{i t}}{|t|^{\alpha}} d t=\frac{\sqrt{\pi} 2^{1-\alpha} \Gamma\left(\frac{1-\alpha}{2}\right)}{\Gamma\left(\frac{\alpha}{2}\right)} .
$$

Using the Parseval equality, we find that

$$
\int_{\mathbb{R}^{2}} \frac{1}{|y-z|^{\alpha}} F(y) F(z) d y d z=\frac{c_{\alpha}}{2 \pi} \int_{\mathbb{R}} \frac{|\hat{F}(\xi)|^{2}}{|\xi|^{1-\alpha}} d \xi .
$$

The right-hand side is finite, because (i) $F \in L^{1}(\mathbb{R})$ so that $\hat{F}(\xi) \in L^{\infty}(\mathbb{R})$, (ii) $F \in$ $L^{1}(\mathbb{R}) \cap L^{\infty}(\mathbb{R})$ so $F \in L^{2}(\mathbb{R})$ and $\hat{F} \in L^{2}(\mathbb{R})$, and (iii) $\alpha \in(0,1)$.

Lemma 3.4. Let $F \in L^{1}(\mathbb{R}) \cap L^{\infty}(\mathbb{R})$ and let the process $\varphi(x)$ be of the form (6) . Then we have:

$$
\lim _{\varepsilon \rightarrow 0} \mathbb{E}\left\{\left(M_{F}^{\varepsilon}-V_{1} M_{F}^{\varepsilon, g}\right)^{2}\right\}=0 .
$$

Proof. We write the square of the integral as a double integral:

$$
\mathbb{E}\left\{\left(M_{F}^{\varepsilon}-V_{1} M_{F}^{\varepsilon, g}\right)^{2}\right\}=\varepsilon^{-\alpha} \int_{\mathbb{R}^{2}} F(y) F(z) Q\left(\frac{y}{\varepsilon}, \frac{z}{\varepsilon}\right) d y d z
$$


where

$$
Q(y, z)=\mathbb{E}\left\{\Phi\left(g_{y}\right) \Phi\left(g_{z}\right)-V_{1} \Phi\left(g_{y}\right) g_{z}-V_{1} g_{y} \Phi\left(g_{z}\right)+V_{1}^{2} g_{y} g_{z}\right\} .
$$

By Lemma 3.2 and (8), there exist $K, T$ such that $|Q(y, z)| \leq K|y-z|^{-2 \alpha}$ for all $|x-y| \geq T$. Besides, $\Phi$ is bounded and $g_{x}$ is square-integrable, so there exists $K$ such that, for all $y, z \in \mathbb{R},|Q(y, z)| \leq K$. We decompose the integration domain $\mathbb{R}^{2}$ into three subdomains $D_{1}, D_{2}$, and $D_{3}$ :

$$
\begin{aligned}
& D_{1}=\left\{(y, z) \in \mathbb{R}^{2},|y-z| \leq T \varepsilon\right\} \\
& D_{2}=\left\{(y, z) \in \mathbb{R}^{2}, T \varepsilon<|y-z| \leq 1\right\}, \\
& D_{3}=\left\{(y, z) \in \mathbb{R}^{2}, 1<|y-z|\right\} .
\end{aligned}
$$

We get the estimates

$$
\begin{aligned}
& \left|\int_{D_{1}} F(y) F(z) Q\left(\frac{y}{\varepsilon}, \frac{z}{\varepsilon}\right) d y d z\right| \leq K \int_{D_{1}}|F(y)||F(z)| d y d z \\
& \leq 2 K \int_{\mathbb{R}} \int_{0}^{T \varepsilon}|F(y+z)| d y|F(z)| d z \\
& \leq 2 K\|F\|_{\infty}\|F\|_{1} T \varepsilon, \\
& \left|\int_{D_{2}} F(y) F(z) Q\left(\frac{y}{\varepsilon}, \frac{z}{\varepsilon}\right) d y d z\right| \leq K \int_{D_{2}}\left|\frac{y}{\varepsilon}-\frac{z}{\varepsilon}\right|^{-2 \alpha}|F(y)| \mid F(z) d y d z \\
& \leq 2 K \varepsilon^{2 \alpha} \int_{\mathbb{R}} \int_{T \varepsilon}^{1} y^{-2 \alpha}|F(y+z)| d y|F(z)| d z \\
& \leq 2 K\|F\|_{1}\|F\|_{\infty} \varepsilon^{2 \alpha} \int_{T \varepsilon}^{1} y^{-2 \alpha} d y \\
& \leq 2 K\|F\|_{1}\|F\|_{\infty}\left\{\begin{array}{l}
\frac{1}{1-2 \alpha} \varepsilon^{2 \alpha} \text { if } \alpha<\frac{1}{2} \\
|\ln (T \varepsilon)| \varepsilon \text { if } \alpha=\frac{1}{2} \\
\frac{T^{1-2 \alpha}}{2 \alpha-1} \varepsilon \text { if } \alpha>\frac{1}{2}
\end{array}\right. \\
& \left|\int_{D_{3}} F(y) F(z) Q\left(\frac{y}{\varepsilon}, \frac{z}{\varepsilon}\right) d y d z\right| \leq K \int_{D_{3}}\left|\frac{y}{\varepsilon}-\frac{z}{\varepsilon}\right|^{-2 \alpha}|F(y)| \mid F(z) d y d z \\
& \leq 2 K \varepsilon^{2 \alpha} \int_{\mathbb{R}} \int_{1}^{\infty} y^{-2 \alpha}|F(y+z)| d y|F(z)| d z \\
& \leq 2 K \varepsilon^{2 \alpha} \int_{\mathbb{R}} \int_{1}^{\infty}|F(y+z)| d y|F(z)| d z \\
& \leq 2 K\|F\|_{1}^{2} \varepsilon^{2 \alpha},
\end{aligned}
$$

which gives the desired result:

$$
\lim _{\varepsilon \rightarrow 0} \varepsilon^{-\alpha}\left|\int_{\mathbb{R}^{2}} F(y) F(z) Q\left(\frac{y}{\varepsilon}, \frac{z}{\varepsilon}\right) d y d z\right|=0 .
$$

The following proposition is now a straightforward corollary of Lemmas 3.3 and 3.4 and the fact that $\kappa=\kappa_{g} V_{1}^{2}$. 
Proposition 3.5. Let $F \in L^{1}(\mathbb{R}) \cap L^{\infty}(\mathbb{R})$ and let the process $\varphi(x)$ be of the form (6).

Then we find that:

$$
\lim _{\varepsilon \rightarrow 0} \mathbb{E}\left\{\left|M_{F}^{\varepsilon}\right|^{2}\right\}=\frac{\kappa 2^{-\alpha} \Gamma\left(\frac{1-\alpha}{2}\right)}{\sqrt{\pi} \Gamma\left(\frac{\alpha}{2}\right)} \int_{\mathbb{R}} \frac{|\hat{F}(\xi)|^{2}}{|\xi|^{1-\alpha}} d \xi
$$

Remark 3.6. The limit of the variance of $M_{F}^{\varepsilon}$ is (34) and the variance of $M^{0}$ is (30). These two expressions are reconciled by using the identity $1-\alpha=2 H-1$ and standard properties of the $\Gamma$ function, namely $\Gamma(H) \Gamma\left(H+\frac{1}{2}\right)=2^{1-2 H} \sqrt{\pi} \Gamma(2 H)$ and $\Gamma(1-H) \Gamma(H)=\pi(\sin (\pi H))^{-1}$. We get

$$
\frac{2^{-\alpha} \Gamma\left(\frac{1-\alpha}{2}\right)}{\sqrt{\pi} \Gamma\left(\frac{\alpha}{2}\right)}=\frac{2^{-2+2 H} \Gamma\left(H-\frac{1}{2}\right)}{\sqrt{\pi} \Gamma(1-H)}=\frac{2^{-2+2 H} \Gamma\left(H+\frac{1}{2}\right)}{\sqrt{\pi}\left(H-\frac{1}{2}\right) \Gamma(1-H)}=\frac{\Gamma(2 H) \sin (\pi H)}{\pi(2 H-1)} .
$$

By (23) this shows that

$$
\frac{2^{-\alpha} \Gamma\left(\frac{1-\alpha}{2}\right)}{\sqrt{\pi} \Gamma\left(\frac{\alpha}{2}\right)} 2 \pi=\frac{1}{H(2 H-1) C(H)^{2}}
$$

and this implies that the variance (30) of $M_{F}^{0}$ is exactly the limit (34) of the variance of $M_{F}^{\varepsilon}$ :

$$
\lim _{\varepsilon \rightarrow 0} \mathbb{E}\left\{\left|M_{F}^{\varepsilon}\right|^{2}\right\}=\mathbb{E}\left\{\left|M_{F}^{0}\right|^{2}\right\}
$$

\subsection{Convergence in distribution}

We can now give the proof of Theorem 3.1

Step 1. The sequence of random variables $M_{F}^{\varepsilon, g}$ defined by (31) converges in distribution as $\varepsilon \rightarrow 0$ to

$$
M_{F}^{0, g}=\sqrt{\frac{\kappa_{g}}{H(2 H-1)}} \int_{\mathbb{R}} F(t) d W_{t}^{H} .
$$

Since the random variable $M_{F}^{\varepsilon, g}$ is a linear transform of a Gaussian process, it has Gaussian distribution. Moreover, its mean is zero. The same statements hold true for $M_{F}^{0, g}$. Therefore, the characteristic functions of $M_{F}^{\varepsilon, g}$ and $M_{F}^{0, g}$ are

$$
\mathbb{E}\left\{e^{i \lambda M_{F}^{\varepsilon, g}}\right\}=\exp \left(-\frac{\lambda^{2}}{2} \mathbb{E}\left\{\left(M_{F}^{\varepsilon, g}\right)^{2}\right\}\right), \quad \mathbb{E}\left\{e^{i \lambda M_{F}^{0, g}}\right\}=\exp \left(-\frac{\lambda^{2}}{2} \mathbb{E}\left\{\left(M_{F}^{0, g}\right)^{2}\right\}\right)
$$

where $\lambda \in \mathbb{R}$. Convergence of the characteristic functions implies that of the distributions [5]. Therefore, it is sufficient to show that the variance of $M_{F}^{\varepsilon, g}$ converges to the variance of $M_{F}^{0, g}$ as $\varepsilon \rightarrow 0$. This follows from Lemma 3.3 .

Step 2: $M_{F}^{\varepsilon}$ converges in distribution to $M_{F}^{0}$ as $\varepsilon \rightarrow 0$.

Let $\lambda \in \mathbb{R}$. Since $M_{F}^{0}=V_{1} M_{F}^{0, g}$, we have

$$
\begin{aligned}
\left|\mathbb{E}\left\{e^{i \lambda M_{F}^{\varepsilon}}\right\}-\mathbb{E}\left\{e^{i \lambda M_{F}^{0}}\right\}\right| \leq & \left|\mathbb{E}\left\{e^{i \lambda M_{F}^{\varepsilon}}\right\}-\mathbb{E}\left\{e^{i \lambda V_{1} M_{F}^{\varepsilon, g}}\right\}\right| \\
& +\left|\mathbb{E}\left\{e^{i \lambda V_{1} M_{F}^{\varepsilon, g}}\right\}-\mathbb{E}\left\{e^{i \lambda V_{1} M_{F}^{0, g}}\right\}\right| .
\end{aligned}
$$


Since $\left|e^{i x}-1\right| \leq|x|$ we can write

$$
\left|\mathbb{E}\left\{e^{i \lambda M_{F}^{\varepsilon}}\right\}-\mathbb{E}\left\{e^{i \lambda V_{1} M_{F}^{\varepsilon, g}}\right\}\right| \leq|\lambda| \mathbb{E}\left\{\left|M_{F}^{\varepsilon}-V_{1} M_{F}^{\varepsilon, g}\right|\right\} \leq|\lambda| \mathbb{E}\left\{\left(M_{F}^{\varepsilon}-V_{1} M_{F}^{\varepsilon, g}\right)^{2}\right\}^{1 / 2},
$$

which goes to zero by the result of Lemma 3.4. This shows that the first term of the right-hand side of (35) converges to 0 as $\varepsilon \rightarrow 0$. The second term of the right-hand side of (35) also converges to zero by the result of Step 1. This completes the proof of Theorem 3.1.

\section{Convergence of random processes}

Let $F_{1}, F_{2}$ be two functions in $L^{\infty}(0,1)$. We consider the random process $M^{\varepsilon}(x)$ defined for any $x \in[0,1]$ by

$$
M^{\varepsilon}(x)=\varepsilon^{-\frac{\alpha}{2}}\left(\int_{0}^{x} F_{1}(t) \varphi^{\varepsilon}(t) d t+x \int_{0}^{1} F_{2}(t) \varphi^{\varepsilon}(t) d t\right) .
$$

With the notation (28) of the previous section, we have

$$
M^{\varepsilon}(x)=M_{F_{x}}^{\varepsilon}=\varepsilon^{-\frac{\alpha}{2}} \int_{\mathbb{R}} F_{x}(t) \varphi^{\varepsilon}(t) d t
$$

where

$$
F_{x}(t)=F_{1}(t) \mathbf{1}_{[0, x]}(t)+x F_{2}(t) \mathbf{1}_{[0,1]}(t)
$$

is indeed a function in $L^{1}(\mathbb{R}) \cap L^{\infty}(\mathbb{R})$.

Theorem 4.1. Let $\varphi$ be a random process of the form (6) and let $F_{1}, F_{2} \in L^{\infty}(0,1)$. Then the random process $M^{\varepsilon}(x)$ defined by (36) converges in distribution as $\varepsilon \rightarrow 0$ in the space of the continuous functions $\mathcal{C}(0,1)$ to the continuous Gaussian process

$$
M^{0}(x)=\sqrt{\frac{\kappa}{H(2 H-1)}} \int_{\mathbb{R}} F_{x}(t) d W_{t}^{H},
$$

where $F_{x}$ is defined by (37) and $W_{t}^{H}$ is a fractional Brownian motion with Hurst index $H=1-\frac{\alpha}{2}$.

The limit random process $M^{0}$ is a Gaussian process with mean zero and autocorrelation function given by

$$
\mathbb{E}\left\{M^{0}(x) M^{0}(y)\right\}=\frac{\kappa}{H(2 H-1)} \times \frac{1}{2 \pi C(H)^{2}} \int_{\mathbb{R}} \frac{\hat{F}_{x}(\xi) \overline{\hat{F}_{y}(\xi)}}{|\xi|^{2 H-1}} d \xi .
$$

The proof of Theorem 4.1 is based on a classical result on the weak convergence of continuous random processes [3]:

Proposition 4.2. Suppose $\left(M^{\varepsilon}\right)_{\varepsilon \in(0,1)}$ are random processes with values in the space of continuous functions $\mathcal{C}(0,1)$ with $M^{\varepsilon}(0)=0$. Then $M^{\varepsilon}$ converges in distribution to $M^{0}$ provided that: 
(i) for any $0 \leq x_{1} \leq \ldots \leq x_{k} \leq 1$, the finite-dimensional distribution $\left(M^{\varepsilon}\left(x_{1}\right), \cdots, M^{\varepsilon}\left(x_{k}\right)\right)$ converges to the distribution $\left(M^{0}\left(x_{1}\right), \ldots, M^{0}\left(x_{k}\right)\right)$ as $\varepsilon \rightarrow 0$.

(ii) $\left(M^{\varepsilon}\right)_{\varepsilon \in(0,1)}$ is a tight sequence of random processes in $\mathcal{C}(0,1)$. A sufficient condition for tightness of $\left(M^{\varepsilon}\right)_{\varepsilon \in(0,1)}$ is the Kolmogorov criterion: $\exists \delta, \beta, C>0$ such that

$$
\mathbb{E}\left\{\left|M^{\varepsilon}(s)-M^{\varepsilon}(t)\right|^{\beta}\right\} \leq C|t-s|^{1+\delta},
$$

uniformly in $\varepsilon, t, s \in(0,1)$.

We split the proof of Theorem 4.1] into two parts: in the next subsection, we prove the point $(i)$, and next, we prove $(i i)$.

\subsection{Convergence of the finite-dimensional distributions}

For the proof of convergence of the finite-dimensional distributions, we want to show that for each set of points $0 \leq x_{1} \leq \cdots \leq x_{k} \leq 1$ and each $\Lambda=\left(\lambda_{1}, \ldots, \lambda_{k}\right) \in \mathbb{R}^{k}$, we have the following convergence result for the characteristic functions:

$$
\mathbb{E}\left\{\exp \left(i \sum_{j=1}^{k} \lambda_{j} M^{\varepsilon}\left(x_{j}\right)\right)\right\} \stackrel{\varepsilon \rightarrow 0}{\longrightarrow} \mathbb{E}\left\{\exp \left(i \sum_{j=1}^{k} \lambda_{j} M^{0}\left(x_{j}\right)\right)\right\} .
$$

Convergence of the characteristic functions implies that of the joint distributions [5]. Now the above characteristic function may be recast as

$$
\mathbb{E}\left\{\exp \left(i \sum_{j=1}^{k} \lambda_{j} M^{\varepsilon}\left(x_{j}\right)\right)\right\}=\mathbb{E}\left\{\exp i\left(\varepsilon^{-\frac{\alpha}{2}} \int_{\mathbb{R}} \varphi^{\varepsilon}(t) F_{\Lambda}(t) d t\right)\right\},
$$

where

$$
F_{\Lambda}(t)=\left(\sum_{j=1}^{k} \lambda_{j} \mathbf{1}_{\left[0, x_{j}\right]}(t)\right) F_{1}(t)+\left(\sum_{j=1}^{k} \lambda_{j} x_{j}\right) \mathbf{1}_{[0,1]}(t) F_{2}(t) .
$$

Since $F_{\Lambda} \in L^{\infty}(\mathbb{R}) \cap L^{1}(\mathbb{R})$ when $F_{1}, F_{2} \in L^{\infty}(0,1)$, we can apply Theorem 3.1 to obtain that:

$$
\mathbb{E}\left\{\exp \left(i \sum_{j=1}^{k} \lambda_{j} M^{\varepsilon}\left(x_{j}\right)\right)\right\} \stackrel{\varepsilon \rightarrow 0}{\longrightarrow} \mathbb{E}\left\{\exp i\left(\sqrt{\frac{\kappa}{H(2 H-1)}} \int_{\mathbb{R}} F_{\Lambda}(t) d W_{t}^{H}\right)\right\},
$$

which in turn establishes (41).

\subsection{Tightness}

It is possible to control the increments of the process $M^{\varepsilon}$, as shown by the following proposition.

Proposition 4.3. There exists $K$ such that, for any $F_{1}, F_{2} \in L^{\infty}(0,1)$ and for any $x, y \in[0,1]$,

$$
\sup _{\varepsilon \in(0,1)} \mathbb{E}\left\{\left|M^{\varepsilon}(y)-M^{\varepsilon}(x)\right|^{2}\right\} \leq K\left(\left\|F_{1}\right\|_{\infty}^{2}|y-x|^{2-\alpha}+\left\|F_{2}\right\|_{\infty}^{2}|y-x|^{2}\right),
$$

where $M^{\varepsilon}$ is defined by (36). 
Proof. The proof is a refinement of the ones of Lemmas 3.3 and 3.4 . We can split the random process $M^{\varepsilon}$ into two components: $M^{\varepsilon}(x)=M^{\varepsilon, 1}(x)+M^{\varepsilon, 2}(x)$, with

$$
M^{\varepsilon, 1}(x)=\varepsilon^{-\frac{\alpha}{2}} \int_{0}^{x} F_{1}(t) \varphi^{\varepsilon}(t) d t, \quad M^{\varepsilon, 2}(x)=x \varepsilon^{-\frac{\alpha}{2}} \int_{0}^{1} F_{2}(t) \varphi^{\varepsilon}(t) d t .
$$

We have

$$
\mathbb{E}\left\{\left|M^{\varepsilon}(y)-M^{\varepsilon}(x)\right|^{2}\right\} \leq 2 \mathbb{E}\left\{\left|M^{\varepsilon, 1}(y)-M^{\varepsilon, 1}(x)\right|^{2}\right\}+2 \mathbb{E}\left\{\left|M^{\varepsilon, 2}(y)-M^{\varepsilon, 2}(x)\right|^{2}\right\} .
$$

The second moment of the increment of $M^{\varepsilon, 2}$ is given by

$$
\mathbb{E}\left\{\left|M^{\varepsilon, 2}(y)-M^{\varepsilon, 2}(x)\right|^{2}\right\}=|x-y|^{2} \varepsilon^{-\alpha} \int_{[0,1]^{2}} R\left(\frac{z-t}{\varepsilon}\right) F_{2}(z) F_{2}(t) d z d t .
$$

Since there exists $K>0$ such that $|R(\tau)| \leq K \tau^{-\alpha}$ for all $\tau$, we have

$$
\begin{aligned}
\varepsilon^{-\alpha} \int_{[0,1]^{2}} R\left(\frac{z-t}{\varepsilon}\right) F_{2}(z) F_{2}(t) d z d t & \leq K \int_{[0,1]^{2}}|z-t|^{-\alpha}\left|F_{2}(z) \| F_{2}(t)\right| d z d t \\
& \leq K\left\|F_{2}\right\|_{\infty}^{2} \int_{-1}^{1}|z|^{-\alpha} d z=\frac{2 K}{1-\alpha}\left\|F_{2}\right\|_{\infty}^{2},
\end{aligned}
$$

which gives the following estimate

$$
\mathbb{E}\left\{\left|M^{\varepsilon, 2}(y)-M^{\varepsilon, 2}(x)\right|^{2}\right\} \leq \frac{2 K}{1-\alpha}\left\|F_{2}\right\|_{\infty}^{2}|x-y|^{2} .
$$

The second moment of the increment of $M^{\varepsilon, 1}$ for $x<y$ is given by

$$
\mathbb{E}\left\{\left|M^{\varepsilon, 1}(y)-M^{\varepsilon, 1}(x)\right|^{2}\right\}=\varepsilon^{-\alpha} \int_{[x, y]^{2}} R\left(\frac{z-t}{\varepsilon}\right) F_{1}(z) F_{1}(t) d z d t .
$$

We distinguish the cases $|y-x| \leq \varepsilon$ and $|y-x| \geq \varepsilon$.

First case. Let us assume that $|y-x| \leq \varepsilon$. Since $R$ is bounded by $V_{2}$, we have

$$
\mathbb{E}\left\{\left|M^{\varepsilon, 1}(y)-M^{\varepsilon, 1}(x)\right|^{2}\right\} \leq V_{2}\left\|F_{1}\right\|_{\infty}^{2} \varepsilon^{-\alpha}|y-x|^{2} .
$$

Since $|y-x| \leq \varepsilon$, this implies

$$
\mathbb{E}\left\{\left|M^{\varepsilon, 1}(y)-M^{\varepsilon, 1}(x)\right|^{2}\right\} \leq V_{2}\left\|F_{1}\right\|_{\infty}^{2}|y-x|^{2-\alpha} .
$$

Second case. Let us assume that $|y-x| \geq \varepsilon$. Since $R$ can be bounded by a power-law function $|R(\tau)| \leq K \tau^{-\alpha}$ we have

$$
\begin{aligned}
\mathbb{E}\left\{\left|M^{\varepsilon, 1}(y)-M^{\varepsilon, 1}(x)\right|^{2}\right\} & \leq K\left\|F_{1}\right\|_{\infty}^{2} \int_{[x, y]^{2}}|z-t|^{-\alpha} d z d t \\
& \leq 2 K\left\|F_{1}\right\|_{\infty}^{2} \int_{x}^{y} \int_{0}^{y-x} t^{-\alpha} d t d z \\
& \leq \frac{2 K}{1-\alpha}\left\|F_{1}\right\|_{\infty}^{2}|y-x|^{2-\alpha}
\end{aligned}
$$

which completes the proof.

This Proposition allows us to get two results.

1) By applying Proposition 4.3 with $F_{2}=0$ and $y=0$, we prove Lemma 2.3.

2) By applying Proposition 4.3, we obtain that the increments of the process $M^{\varepsilon}$ satisfy the Kolmogorov criterion (40) with $\beta=2$ and $\delta=1-\alpha>0$. This gives the tightness of the family of processes $M^{\varepsilon}$ in the space $\mathcal{C}(0,1)$. 


\subsection{Proof of Theorem 2.5}

We can now give the proof of Theorem 2.5. The error term can be written in the form

$$
\varepsilon^{-\frac{\alpha}{2}}\left(u^{\varepsilon}(x)-\bar{u}(x)\right)=\varepsilon^{-\frac{\alpha}{2}}\left(\int_{0}^{x} F_{1}(t) \varphi^{\varepsilon}(t) d t+x \int_{0}^{1} F_{2}(t) \varphi^{\varepsilon}(t) d t\right)+\widetilde{r}^{\varepsilon}(x),
$$

where $F_{1}(t)=c^{*}-F(t), F_{2}(t)=F(t)-\int_{0}^{1} F(z) d z-a^{*} q$, and $\widetilde{r}^{\varepsilon}(x)=\varepsilon^{-\alpha / 2}\left[r^{\varepsilon}(x)+\right.$ $\left.\rho^{\varepsilon} a^{*-1} x\right]$. The first term of the right-hand side is of the form (36). Therefore, by applying Theorem 4.1, we get that this process converges in distribution in $\mathcal{C}(0,1)$ to the limit process (19). It remains to show that the random process $\widetilde{r}^{\varepsilon}(x)$ converges as $\varepsilon \rightarrow 0$ to zero in $\mathcal{C}(0,1)$ in probability.

We have

$$
\mathbb{E}\left\{\left|\widetilde{r}^{\varepsilon}(x)-\widetilde{r}^{\varepsilon}(y)\right|^{2}\right\} \leq 2 \varepsilon^{-\alpha} \mathbb{E}\left\{\left|r^{\varepsilon}(x)-r^{\varepsilon}(y)\right|^{2}\right\}+2 a^{*-2} \varepsilon^{-\alpha} \mathbb{E}\left\{\left|\rho^{\varepsilon}\right|^{2}\right\}|x-y|^{2},
$$

From the expression (18) of $r^{\varepsilon}$, and the fact that $c^{\varepsilon}$ can be bounded uniformly in $\varepsilon$ by a constant $c_{0}$, we get

$$
\varepsilon^{-\alpha} \mathbb{E}\left\{\left|r^{\varepsilon}(x)-r^{\varepsilon}(y)\right|^{2}\right\} \leq 2 \varepsilon^{-\alpha} c_{0} \mathbb{E}\left\{\left|\int_{x}^{y} \varphi^{\varepsilon}(t) d t\right|^{2}\right\} .
$$

Upon applying Proposition 4.3, we obtain that there exists $K>0$ such that

$$
\varepsilon^{-\alpha} \mathbb{E}\left\{\left|r^{\varepsilon}(x)-r^{\varepsilon}(y)\right|^{2}\right\} \leq K|x-y|^{2-\alpha} .
$$

Besides, since $\rho^{\varepsilon}$ can be bounded uniformly in $\varepsilon$ by a constant $\rho_{0}$, we have $\mathbb{E}\left\{\left|\rho^{\varepsilon}\right|^{2}\right\} \leq$ $\rho_{0} \mathbb{E}\left\{\left|\rho^{\varepsilon}\right|\right\} \leq K \varepsilon^{\alpha}$ for some $K>0$. Therefore, we have established that there exists $K>0$ such that

$$
\mathbb{E}\left\{\left|\widetilde{r}^{\varepsilon}(x)-\widetilde{r}^{\varepsilon}(y)\right|^{2}\right\} \leq K|x-y|^{2-\alpha},
$$

uniformly in $\varepsilon, x, y$. This shows that $\widetilde{r}^{\varepsilon}(x)$ is a tight sequence in the space $\mathcal{C}(0,1)$ by the Kolmogorov criterion (40). Furthermore, the finite-dimensional distributions of $\widetilde{r}^{\varepsilon}(x)$ converges to zero because

$$
\sup _{x \in[0,1]} \mathbb{E}\left\{\left|\widetilde{r}_{\varepsilon}(x)\right|\right\} \stackrel{\varepsilon \rightarrow 0}{\longrightarrow} 0
$$

by (15) and (17). Proposition 4.2 then shows that $\widetilde{r}^{\varepsilon}(x)$ converges to zero in distribution in $\mathcal{C}(0,1)$. Since the limit is deterministic, the convergence actually holds true in probability.

\section{Numerical results for Theorem 2.6}

In this section, we numerically study the convergence of the error term in the case where $F=0, q=1$, and the driving process $\varphi(x)$ has an integrable autocorrelation function. The solutions of the random elliptic equation (11) and of the homogenized equation (2) are given by

$$
u_{\varepsilon}(x)=\frac{1}{\int_{0}^{1} \frac{1}{a_{\varepsilon}} d y} \int_{0}^{x} \frac{1}{a_{\varepsilon}} d y \quad ; \quad \bar{u}(x)=x .
$$


Using the decomposition $\varphi_{\varepsilon}=\frac{1}{a_{\varepsilon}}-\frac{1}{a_{*}}$ and assuming that $a_{*}=1$, we have

$$
u_{\varepsilon}(x)=\frac{x+\int_{0}^{x} \varphi_{\varepsilon} d y}{1+\int_{0}^{1} \varphi_{\varepsilon} d y} .
$$

We study the the convergence of the process at the point $x=\frac{1}{2}$, where we have

$$
u_{\varepsilon}\left(\frac{1}{2}\right)=\frac{\frac{1}{2}+\int_{0}^{\frac{1}{2}} \varphi_{\varepsilon} d y}{1+\int_{0}^{1} \varphi_{\varepsilon} d y} \stackrel{\varepsilon \rightarrow 0}{\longrightarrow} \frac{1}{2}=\bar{u}\left(\frac{1}{2}\right) .
$$

\subsection{Generation of the driving process}

We carry out numerical simulations in the case where the random process $\varphi(x)$ is of the form $\Phi\left(g_{x}\right)$ with $g_{x}$ a stationary Ornstein-Uhlenbeck process and $\Phi(x)=\frac{1}{2} \operatorname{sgn}(x)$ (see Figure 11). This is a simple model for a two-component random medium. [5]

The Ornstein-Uhlenbeck process is the solution of the stochastic differential equation

$$
d g_{x}=-g_{x} d x+\sqrt{2} d W_{x},
$$

where $W_{x}$ is a standard Brownian motion. If we suppose that $g_{0}$ is a Gaussian random variable with mean 0 and variance 1 independent of the driving Brownian motion, then $\left(g_{x}\right)_{x \geq 0}$ is a stationary, mean zero, Gaussian process with the autocorrelation function $\mathbb{E}\left\{g_{x} g_{x+\tau}\right\}=\exp (-|\tau|)$. Moreover, it is a Markovian process, which makes it easy to simulate a realization of the Ornstein-Uhlenbeck process $\left(g_{k \Delta x}\right)_{k \geq 0}$ sampled at times $(k \Delta x)_{k \geq 0}$ by the following recursive procedure:

- $g_{0}=G_{0}$,

- $g_{(k+1) \Delta x}=e^{-\Delta x} g_{k \Delta x}+\sqrt{1-\mathrm{e}^{-2 \Delta x}} G_{k+1}$,

where $\left(G_{k}\right)_{k \geq 0}$ is a sequence of independent and identically distributed Gaussian random variables with mean 0 and variance 1 . Note that the simulation is exact independently of the value of the grid step $\Delta x$.

Lemma 5.1. If $g_{x}$ is the stationary Ornstein-Uhlenbeck process and $\varphi(x)=\frac{1}{2} \operatorname{sgn}\left(g_{x}\right)$, then $\varphi(x)$ is a stationary, mean zero, random process with the autocorrelation function

$$
R(\tau)=\mathbb{E}\{\varphi(x+\tau) \varphi(x)\}=\frac{1}{4}\left(1-\frac{2}{\pi} \arctan \left(\sqrt{\mathrm{e}^{2|\tau|}-1}\right)\right) .
$$

Proof. Since $g \mapsto \operatorname{sgn}(g)$ is an odd function, it is obvious that $\varphi(x)$ has mean zero. Denoting $a_{\tau}=e^{-|\tau|}$ and $b_{\tau}=\sqrt{1-e^{-2|\tau|}}$, the autocorrelation function of $\varphi(x)$ can be 


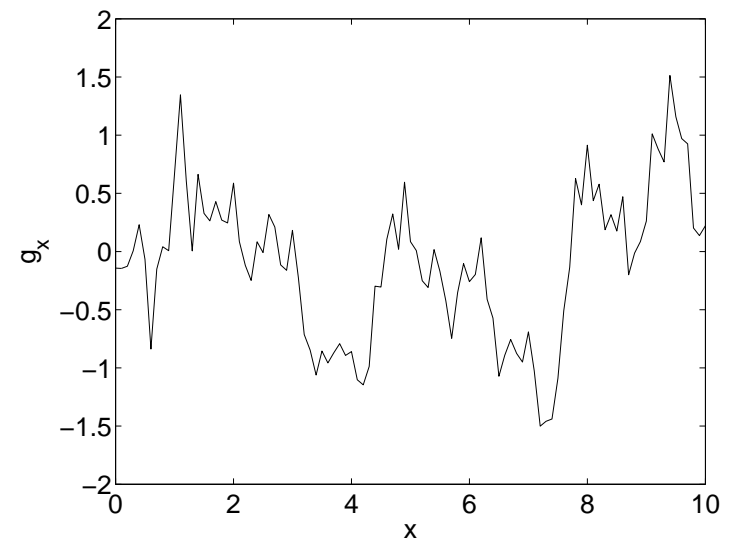

(a)

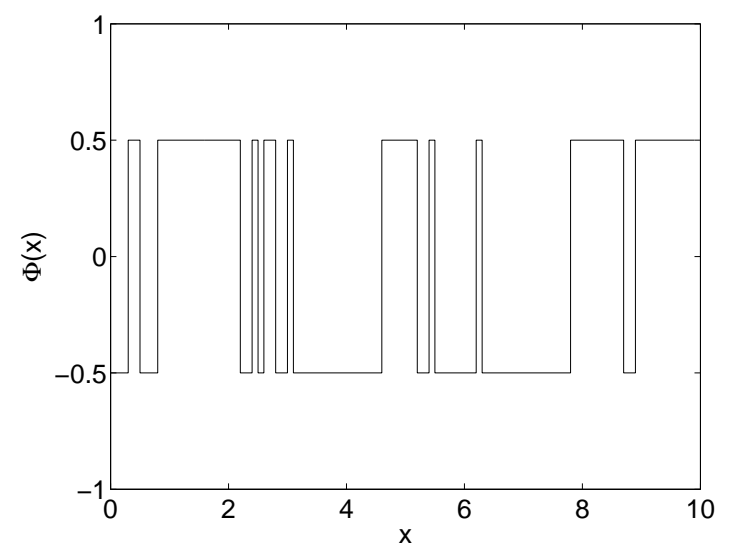

(b)

Figure 1: Simulation of the Ornstein-Uhlenbeck process $g_{x}$ (picture (a)) and the induced bounded process $\varphi(x)=\frac{1}{2} \operatorname{sgn}\left(g_{x}\right)$ (picture (b)).

computed as follows:

$$
\begin{aligned}
R(\tau) & =\mathbb{E}\left\{\Phi\left(g_{0}\right) \Phi\left(g_{\tau}\right)\right\}=\frac{1}{4} \mathbb{E}\left\{\operatorname{sgn}\left(g_{0}\right) \operatorname{sgn}\left(g_{\tau}\right)\right\} \\
& =\frac{1}{4} \frac{1}{2 \pi} \int_{\mathbb{R}^{2}} \operatorname{sgn}(x) \operatorname{sgn}\left(a_{\tau} x+b_{\tau} y\right) \mathrm{e}^{-\frac{x^{2}+y^{2}}{2}} d x d y \\
& =\frac{1}{4} \frac{2}{2 \pi} \int_{\mathbb{R}^{+} \times \mathbb{R}} \operatorname{sgn}(x) \operatorname{sgn}\left(a_{\tau} x+b_{\tau} y\right) \mathrm{e}^{-\frac{x^{2}+y^{2}}{2}} d x d y \\
& =\frac{1}{4 \pi} \int_{0}^{\infty} \int_{\theta=-\pi / 2}^{-\pi / 2+\theta_{\tau}}(-1) \rho \mathrm{e}^{-\frac{\rho^{2}}{2}} d \theta d \rho+\frac{1}{4 \pi} \int_{0}^{\infty} \int_{\theta=\theta_{\tau}}^{\pi / 2} 1 \rho \mathrm{e}^{-\frac{\rho^{2}}{2}} d \theta d \rho \\
& =\frac{1}{4 \pi}\left[-\theta_{\tau}+\left(\pi-\theta_{\tau}\right)\right]=\frac{1}{4}\left(1-\frac{2}{\pi} \theta_{\tau}\right),
\end{aligned}
$$

with $\theta_{\tau}=\arctan \left(\frac{b_{\tau}}{a_{\tau}}\right)=\arctan \left(\sqrt{\mathrm{e}^{2|\tau|}-1}\right)$.

\subsection{Convergence of the corrector}

We now study the convergence of $u_{\varepsilon}\left(\frac{1}{2}\right)$ to $\bar{u}\left(\frac{1}{2}\right)$. The value of the integral $\int_{0}^{1} F(s) \varphi_{\varepsilon}(s) d s$ is approximated by the standard quadrature formula

$$
\int_{0}^{1} F(s) \varphi_{\varepsilon}(s) d s=\int_{0}^{1} F(s) \varphi\left(\frac{s}{\varepsilon}\right) d s=\varepsilon \int_{0}^{1 / \varepsilon} F(\varepsilon y) \varphi(y) d y \approx \varepsilon \sum_{i=0}^{n} F(i \varepsilon \Delta x) \varphi(i \Delta x) \Delta x,
$$

with $n=[1 /(\varepsilon \Delta x)]$ and $\Delta x=0.1$ in our simulations.

We first estimate the convergence order of the variance of $\left(u_{\varepsilon}-\bar{u}\right)\left(\frac{1}{2}\right)$ when $\varepsilon \rightarrow 0$. The following values are given to $\varepsilon$ :

$$
\varepsilon \in\{0.0001,0.0002,0.0005,0.001,0.002,0.005,0.01,0.05,0.1\} .
$$




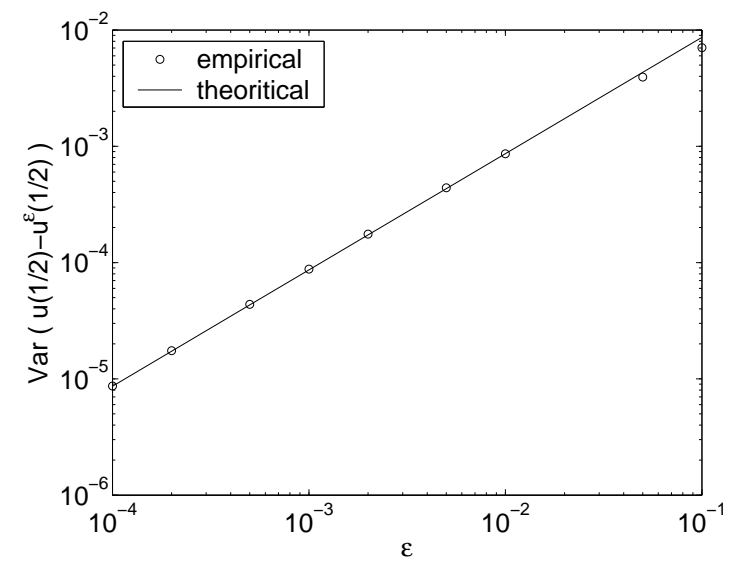

(a)

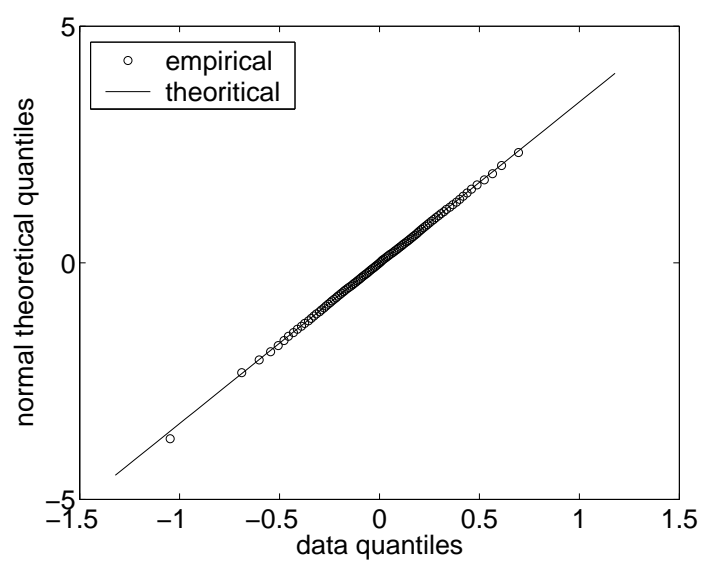

(b)

Figure 2: Picture (a): Variance of $\left(u_{\varepsilon}-u\right)\left(\frac{1}{2}\right)$ as a function of $\varepsilon$, in $\log -\log$ scale. The convergence rate of the variance in $\log \log$ scale has a slope equal to one, which proves that the convergence is proportional to $\varepsilon$. Picture (b): Normal QQ plot for the distribution of $\varepsilon^{-\frac{1}{2}}\left(u^{\varepsilon}-\bar{u}\right)\left(\frac{1}{2}\right)$ with $\varepsilon=0.0001$, which confirms the Gaussian behavior of the error.

For each $\varepsilon$, we perform $10^{4}$ simulations and compute the empirical variance. The results are shown in Figure 2 a. The asymptotic theory predicts that the convergence is linear in $\varepsilon$ :

$$
\operatorname{Var}\left\{u_{\varepsilon}\left(\frac{1}{2}\right)-\bar{u}\left(\frac{1}{2}\right)\right\}=\sigma^{2} \varepsilon+o(\varepsilon), \quad \sigma^{2}=2 a_{*} \int_{0}^{\infty} R(\tau) d \tau \approx 0.0865
$$

The computation of a linear regression of the empirical variance with respect to $\varepsilon$, with the two, three, etc.. first points give $0.0865,0.0875,0.0870$, which is different from the theoretical prediction by less than $1 \%$.

We now check the convergence in law of $\frac{1}{\sqrt{\varepsilon}}\left(u_{\varepsilon}\left(\frac{1}{2}\right)-\bar{u}\left(\frac{1}{2}\right)\right)$. Theorem 2.6 predicts that

$$
\frac{1}{\sqrt{\varepsilon}}\left(u_{\varepsilon}(x)-\bar{u}(x)\right) \stackrel{\text { law }}{\longrightarrow}\left(2 \int_{0}^{\infty} R(\tau) d \tau\right)^{1 / 2} \mathcal{U}(x),
$$

with $\mathcal{U}(x)=a_{*} W_{x}-a_{*} x W_{1}$, so that in our case

$$
\frac{1}{\sqrt{\varepsilon}}\left(u_{\varepsilon}\left(\frac{1}{2}\right)-\bar{u}\left(\frac{1}{2}\right)\right) \stackrel{\text { law }}{\longrightarrow} G
$$

where $G$ is a Gaussian random variable with mean zero and variance

$$
\sigma^{2}=2 \int_{0}^{\infty} R(\tau) d \tau \operatorname{Var}\left\{\mathcal{U}\left(\frac{1}{2}\right)\right\}=2 a_{*} \int_{0}^{\infty} R(\tau) d \tau \approx 0.0865
$$

In Figure 2 $\mathrm{b}$, we compare the distribution of $\frac{1}{\sqrt{\varepsilon}}\left(u_{\varepsilon}\left(\frac{1}{2}\right)-\bar{u}\left(\frac{1}{2}\right)\right)$ for $\varepsilon=10^{-4}$ with the one of $G$ by plotting the normal QQ plot which shows perfect agreement (a normal QQ plot is a scatter-plot with the quantiles of the sample on the horizontal axis and the expected normal quantiles on the vertical axis). 


\section{Numerical results for Theorem 2.5}

\subsection{Generation of the driving process}

To test the result of Theorem 2.5, we need to generate a Gaussian process with a heavy tail. We choose to generate the increments of a fractional Brownian motion: $g_{x}=W_{x+1}^{H}-W_{x}^{H}$. Since fractional Brownian motion is not a Markovian process, it cannot be generated iteratively. Many different methods have been developed to simulate fractional Brownian motions based on integral representations in terms of standard Brownian motions, spectral representations, or wavelet decompositions (see the review [1]). In this paper we use the Choleski method because it is the simplest method to implement. It is based on the following facts:

1) the fractional Brownian motion $W_{x}^{H}$ and the process $g_{x}$ are Gaussian processes,

2) the autocorrelation function of the fractional Brownian motion is known (see (21)), so that it is possible to calculate the covariance matrix $C$ of the Gaussian vector $\left(g_{k \Delta x}\right)_{k=0, \ldots, N}$,

3) if $X$ is a vector of independent and identically distributed random variables with Gaussian distribution, mean 0 , and variance 1 , then $M X$ is a mean zero Gaussian vector with covariance matrix $M M^{T}$.

The Choleski method consists in

1) computing a square root $\sqrt{C}$ of the covariance matrix $C$ of the Gaussian vector $\left(g_{k \Delta x}\right)_{k=0, \ldots, N}$,

2) generating a vector $X$ of $N+1$ independent and identically distributed Gaussian random variables with mean 0 and variance 1 ,

3) computing the vector $\sqrt{C} X$.

This method is exact, in the sense that the simulated vector $\sqrt{C} X$ has the distribution of $\left(g_{k \Delta x}\right)_{k=0, \ldots, N}$, whatever the grid step $\Delta x$ may be. The method is, however, computationally expensive. In fact, only the computation of the square root of the matrix $C$ is costly. Once this computation has been carried out, it is straightforward to generate a sequence of independent and identically distributed random vectors with the distribution of $\left(g_{k \Delta x}\right)_{k=0, \ldots, N}$.

We apply the Choleski method to generate $10^{5}$ realizations of the vector $\left(g_{k \Delta x}\right)_{k=0, \ldots, N}$ with $\Delta x=1$ and $N=2000$. The Hurst parameter is equal to 0.8. The empirical autocorrelation function is shown in Figure 3 and compared with its theoretical asymptotic behavior $\tau \mapsto H(2 H-1) \tau^{2 H-2}[\tau \rightarrow \infty]$. When $\tau$ becomes large, the fluctuations become large compared to $R(\tau)$ because $R(\tau) \rightarrow 0$. A linear regression made on the interval $[10,100]$ gives the power law fit $K t^{\beta}$, with $K=0.4901$ and $\beta=0.3964$, which is in agreement with the theoretical values $K=0.48$ and $\beta=0.4$.

We suppose that the random medium is described by the stationary random process

$$
\frac{1}{a(x)}=\frac{9}{2}+\frac{8}{\pi} \arctan \left(g_{x}\right)
$$

The asymptotic behavior of its autocorrelation function is theoretically given by (11) with

$$
V_{1}=\frac{1}{\sqrt{2 \pi}} \frac{8}{\pi} \int_{-\infty}^{+\infty} x \arctan (x) \mathrm{e}^{-\frac{x^{2}}{2}} d x \approx 1.6694
$$




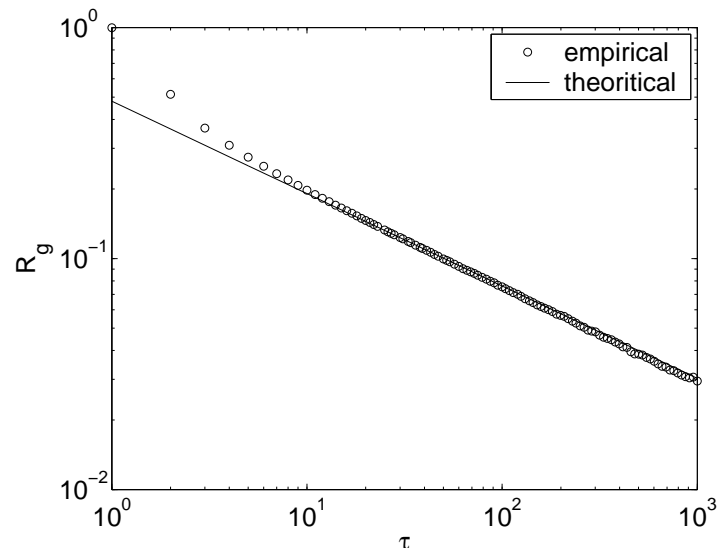

(a)

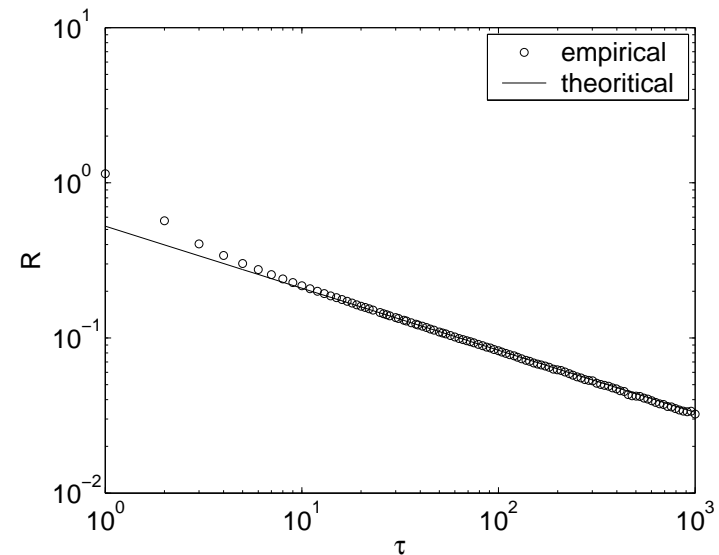

(b)

Figure 3: A series of $10^{5}$ numerical simulations of the vector $\left(g_{k \Delta x}\right)_{k=0, \ldots, N}$ is carried out in the case where $g_{x}=W_{x+1}^{H}-W_{x}^{H}, H=0.8, N=2000$, and $\Delta x=1$. Picture (a): The empirical autocorrelation of $g_{x}$ is compared with the theoretical asymptotic behavior $\tau \mapsto H(2 H-1) \tau^{2 H-2}$. Picture (b): The empirical autocorrelation of $\varphi(x)$ is compared with the theoretical asymptotic behavior $\tau \mapsto V_{1}^{2} H(2 H-1) \tau^{2 H-2}$.

The empirical autocorrelation function of the process determined by a series of $10^{5}$ experiments is shown in Figure 3, where we observe that the theoretical and empirical curves agree very well.

\subsection{Convergence of the corrector}

We now study the convergence of the solution of the homogenization problem (II) as $\varepsilon \rightarrow 0$. We choose $F(x)=x^{2}$ and $q=1$. For $a(x)$ given by (44), we find that $a^{\star}=\frac{2}{9}$. A solution obtained with a particular realization of the random process with $\varepsilon=0.0033$ is shown in Figure 4 and compared with the theoretical solution of the homogenized problem.

We estimate the order of convergence of the variance of the corrector $\left(u_{\varepsilon}-u\right)\left(\frac{1}{2}\right)$ when $\varepsilon \rightarrow 0$. The following values are given to $\varepsilon$ :

$$
\varepsilon \in\{0.0033,0.0017,0.0011,0.00091,0.00077,0.00062,0.0004\} .
$$

For each value of $\varepsilon$, we run $10^{4}$ numerical experiments, compute the empirical variance of the corrector, and compare with the asymptotic theoretical variance predicted by Theorem 2.5.

$$
\operatorname{Var}\left\{u_{\varepsilon}\left(\frac{1}{2}\right)-\bar{u}\left(\frac{1}{2}\right)\right\}=\sigma_{H}^{2} \varepsilon^{2-2 H}+o\left(\varepsilon^{2-2 H}\right)
$$

with $2-2 H=0.4$ and

$$
\sigma_{H}^{2}=\operatorname{Var}\left(\sqrt{\frac{\kappa}{H(2 H-1)}} \int_{\mathbb{R}} K\left(\frac{1}{2}, t\right) d W_{t}^{H}\right) \approx 0.0553 .
$$

The results are presented in Figure $5 \mathrm{~b}$ and show good agreement. More quantitatively, a linear regression of the logarithm of the empirical variance of the error with respect 


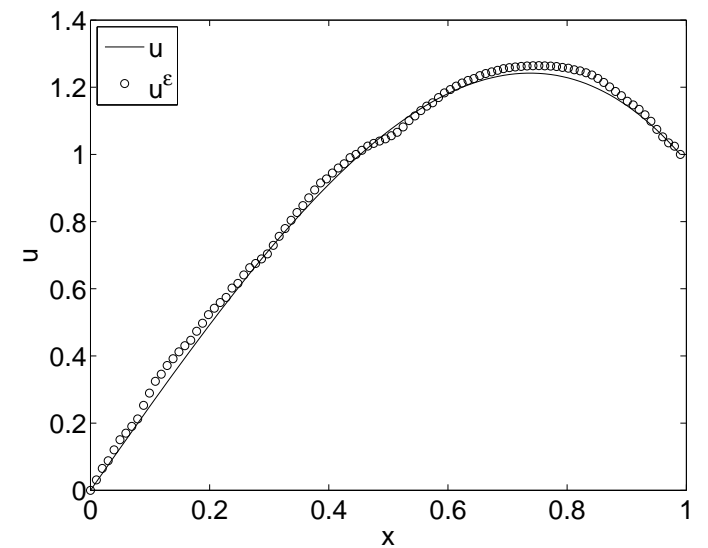

(a)

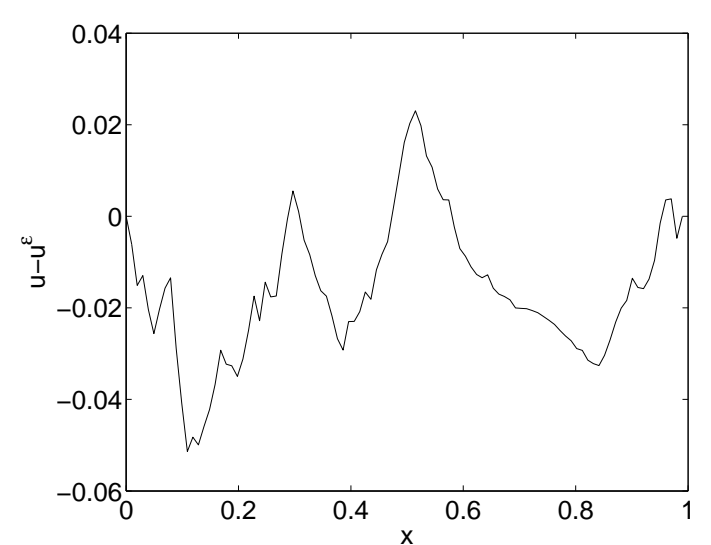

(b)

Figure 4: Picture (a) compares the homogenized solution (solid line) with the solution of (11) obtained for $\varepsilon=0.0033$ and for a particular realization of the random process $\varphi$ (circles). Picture (b) plots the difference between $u^{\varepsilon}$ and $\bar{u}$.

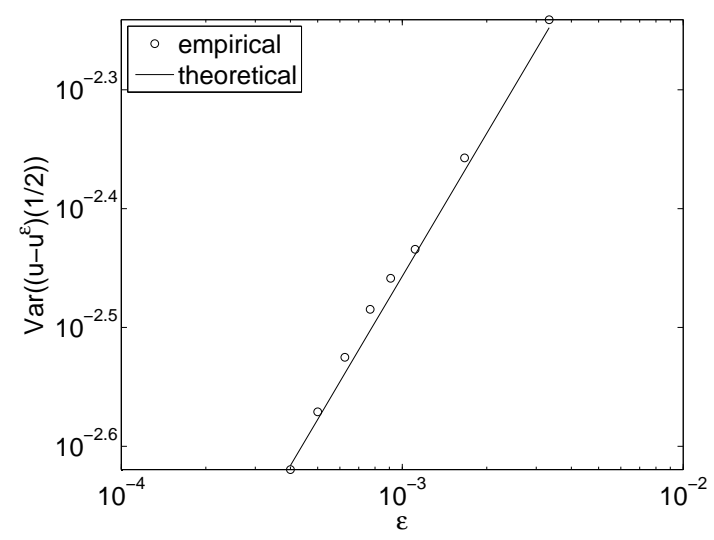

(a)

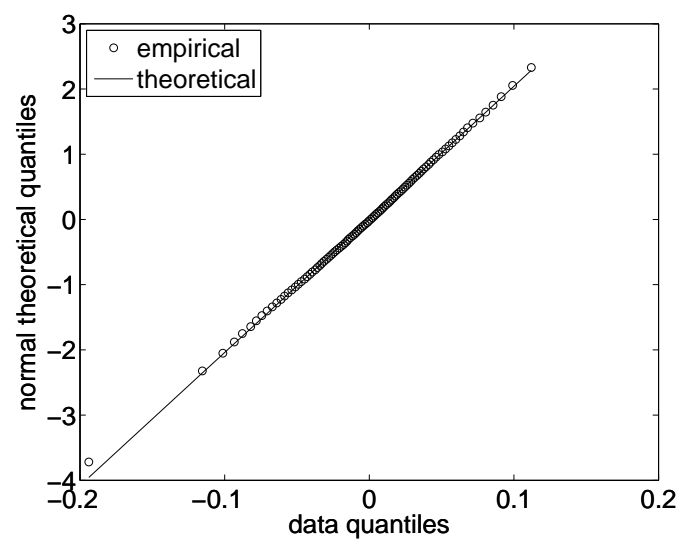

(b)

Figure 5: Picture (a): Empirical variance of $\left(u_{\varepsilon}-\bar{u}\right)\left(\frac{1}{2}\right)$ as a function of $\varepsilon$, in $\log$ log scale (circles), compared with the asymptotic theoretical variance (46) predicted by Theorem [2.5. Picture (b): Normal QQ plot for $\left(u^{\varepsilon}-\bar{u}\right)\left(\frac{1}{2}\right)$ with $\varepsilon=0.0004$, which confirms the Gaussian behavior of the corrector term. 
to $\log \varepsilon$ gives:

$$
\operatorname{Var}\left\{u^{\varepsilon}\left(\frac{1}{2}\right)-\bar{u}\left(\frac{1}{2}\right)\right\} \approx 0.0581 \varepsilon^{0.4041}
$$

which agrees very well with (46). Finally, we can check that the distribution of the limit process is Gaussian by observing that the normal QQ plot in Figure 5 b is indeed a straight line.

\section{Conclusions}

We have shown that the corrector to homogenization, i.e., the difference between the random solution to an elliptic equation with random coefficients and the deterministic solution to the appropriately homogenized elliptic equation, strongly depends on the statistics of the random medium. When the correlation function of the random diffusion coefficient is integrable, such a corrector is of order $\sqrt{\varepsilon}$, where $\varepsilon$ measures the correlation length of the random medium. When the correlation function behaves like $\tau^{-\alpha}$, which measures long-memory effects, then the difference becomes of order $\varepsilon^{\frac{\alpha}{2}}$ for $0<\alpha<1$. The corrector to the homogenized solution may thus be arbitrarily large asymptotically for very small values of $\alpha$, which corresponds to pronounced long-memory effects.

Moreover, up to smaller terms of order $\varepsilon^{\alpha}$, the corrector to homogenization is a centered Gaussian process, which may conveniently and explicitly be written as a stochastic integral with respect to fractional Brownian motion. Such a random behavior of the corrector may provide an accurate quantitative model for the statistical instability (i.e., the dependency with respect to the specific realization of the random medium) of practical and experimental measurements. This central-limit-type behavior may be extremely difficult and costly to adequately capture by numerical simulations of the elliptic equation with random coefficients because of the very large number of random variables involved in the modeling of a random medium with a small correlation length $(\varepsilon \ll 1)$.

The results presented in this paper are based on the explicit integral representation (3) of the solution to the one-dimensional elliptic equation (11). Such formulas are not available in higher space dimensions. Although we are tempted to believe that similar behaviors will remain valid in higher space dimensions, namely that long-memory effects will trigger large random corrections to homogenization, this remains a conjecture at present.

\section{References}

[1] J.-M. Bardet, G. Lang, G. Oppenheim, A. Philippe, and M. S. Taqqu, Generators of the long-range dependence processes: a survey, in Theory and applications of long-range dependence, P. Doukhan, G. Oppenheim, and M. S. Taqqu, editors, Birkhauser (2003), pp. 579-624.

[2] A. Bensoussan, J.-L. Lions, and G. C. Papanicolaou, Boundary layers and homogenization of transport processes, Res. Inst. Math. Sci., Kyoto Univ. 15 (1979), $53-157$.

[3] P. Billingsley, Convergence of Probability Measures, Wiley, New York, 1999. 
[4] A. Bourgeat And A. Piatnitski, Estimates in probability of the residual between the random and the homogenized solutions of one-dimensional second-order operator, Asympt. Anal. 21 (1999), 303-315.

[5] L. Breiman, Probability, Classics in Applied Mathematics, SIAM, Philadelphia, 1992.

[6] V. V. Jikov, S. M. Kozlov, and O. A. Oleinik, Homogenization of differential operators and integral functionals, Springer, New York, 1994.

[7] S. M. KozLov, The averaging of random operators, Math. USSR Sb. 109 (1979), 188-202.

[8] H. J. Kushner, Approximation and weak convergence method for random processes, with applications to stochastic systems theory, MIT Press, Cambridge, 1984.

[9] R. MARTY, Asymptotic behavior of differential equations driven by periodic and random processes with slowly decaying correlations, ESAIM: Probability and Statistics 9 (2005), 165-184.

[10] G. C. Papanicolaou And S. R. S. Varadhan, Boundary value problems with rapidly oscillating random coefficients, in Random fields, Vol. I, II (Esztergom, 1979), Colloq. Math. Soc. János Bolyai, 27, North Holland, New York, 1981, pp. 835-873.

[11] V. Pipiras And M. S. Taqqu, Integration questions related to fractional Brownian motion, Probab. Theor. Related Fields 118 (2000), 251-291.

[12] M. S. TAQQU, Weak convergence to fractional Brownian motion and to the Rosenblatt process, Z. Wahrscheinlichkeitstheorie verw. Geb. 31 (1975), 287-302; Convergence of integrated processes of arbitrary Hermite rank, Z. Wahrscheinlichkeitstheorie verw. Geb. 50 (1979), 53-83.

[13] V. V. YurinskiI, Averaging of symmetric diffusion in a random medium, Siberian Math. J. 4 (1986), 603-613. English translation of: Sibirsk. Mat. Zh. 27 (1986), 167180 (Russian). 